\title{
Should smoke exposure be considered an etiologic factor for cardiovascular disease?
}

"...cigarette smoking itself is able to cause a toxic disease whose etiologic factor should be considered the tobacco with its chemical compounds."

First draft submitted: 25 January 2016; Accepted for publication: 25 January 2016; Published online: 28 January 2016

Keywords: blood vessels $\bullet$ etiologic factor $\bullet$ heart $\bullet$ smoking

It is commonly believed and nearly completely accepted that cigarette smoking is a major cardiovascular risk factor for the heart and blood vessels because of the effects of some compounds, primarily nicotine and carbon monoxide, which are able to harm the cardiovascular system [1-5]. As a result of this, even passive smoking was included by the American Heart Association in their group of cardiovascular risk factors for both adolescents and adults [2].

Ischemic heart disease and endothelial dysfunction are the main effects observed as a result of both types of smoking - active and passive smoke exposure [6-9]. These changes have been clearly documented by clinical, pathological and experimental findings and, although they are of a different degree and extent in various reports, are reproducible in the same standardized procedures [10-12]. Functional, but initially reversible, and structural alterations [11,12], which become over the years firm lesions [13,14], may often be seen as a consequence of nicotine and carbon monoxide activity.

Briefly, impaired endothelium-dependent vasodilation due to a decrease in nitric oxide availability; reduced tolerance to exercise in both healthy individuals and individuals suffering from ischemic heart disease; as well as enhanced heart rate and systolic blood pressure due to sympathetic nervous system stimulation, carboxyhemoglobin and increased catecholamine release, are the transient and functional responses observed during acute exposure to smoking. On the other hand, chronic exposure to tobacco smoke causes well-established pathological disorders, typically due to carbon monoxide, consisting of myocardial lesions related to ischemic heart disease, and smoke cardiomyopathy with intracellular and ultrastructural alterations, primarily identified in experimental animals [14]. These lead to heart failure of various degrees, which mainly depends on the duration of the exposure. Moreover, vascular alterations of a degenerative type, which develop into atherosclerosis and its complications, affect primarily coronary, carotid and cerebral arteries. In addition, it is worth noting that microcirculation, because of the structure of resistance arteries, shows arteriosclerosis with enhanced vascular tone, which is the result of the anatomical lesions in the artery wall characterized by elastic fiber fragmentation and collagen deposits [15]. Increased arterial stiffness has also been documented in heavy smokers [16].

Therefore, it is worth noting that six markers often may change as a consequence of smoking exposure: endothelium with endothelial dysfunction; artery wall with lesions of different degrees including up to atherosclerotic plaque formation; blood pressure, which increases systolic values; heart rate, which closely follows blood pressure characteristics; myocardial function impairment and structural alterations of myocardial fibers leading up to necrosis.

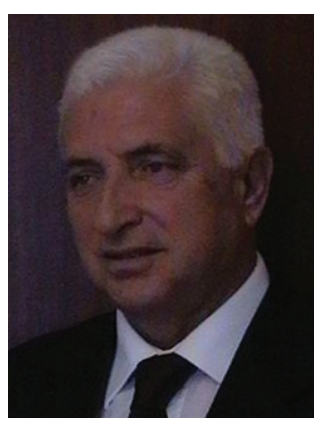

Aurelio Leone ${ }^{1}$ Fellow of the Royal Society for Promotion of Health (FRSPH), UK ${ }^{2}$ Fellow of the American Society of Hypertension, USA Via Provinciale 27, 19030 Castelnuovo Magra, Italy

Tel.: +393472272215 reliol@libero.it 
Smoking compounds exert cardiovascular damage by direct and mediated mechanisms involving sympathetic and adrenergic stimulation, increased blood carboxyhemoglobin concentrations and cellular hypoxia [17].

By carefully analyzing these data, a fundamental concept emerges: cigarette smoking always causes the same damage to the heart and blood vessels in all subjects exposed, although the characteristics and type depend on various factors, primarily duration of the exposure to smoking toxics. In addition, there is evidence that a strongly adverse relationship, which is undoubtedly above a mere risk, exists between smoking and the cardiovascular system.

A risk factor [18] is associated with the underlying disease with a statistical, but probabilistic relation. The risk is expressed through a statistical estimate that, as it becomes closer to $100 \%$ - which, however, never can be reached - as a higher occurrence of the event related is demonstrated. Removing the risk factor can dramatically reduce, but not completely abolish the rate of the underlying disease since other, not always well-assessed conditions often contribute to its maintenance.

On the contrary, an etiologic factor [19] recognizes a link of cause-effect with the underlying disease, taking into account, however, that the occurrence of the disease is dependent on the power and potential charge of the pathogenic agent. When it reaches levels able to determine a disease, this event will always be seen with the same, even if of a different degree, type of alterations. So for example, when, in an infective disease with known etiology the causative micro-organism reaches a bacterial charge able to develop the disease, it always will occur.

It is worth noting, as aforementioned, that cigarette smoking should be considered an etiologic rather than

\section{References}

1 Leone A. Cardiovascular damage from smoking: a fact or belief? Int. J. Cardiol. 38, 113-117 (1993).

2 Glantz SA, Parmley WW. Passive smoking and heart disease: mechanisms and risk. JAMA 273(13), 1047-1053 (1995).

3 McGill HC Jr. The cardiovascular pathology of smoking. Am. Heart J. 115, 250-257 (1988)

4 Auerbach O, Carter HW, Garfinkel L, Hammond EC. Cigarette smoking and coronary heart disease, a macroscopic and microscopic study. Chest 70, 697-705 (1976).

5 Leone A. Biochemical markers of cardiovascular damage from tobacco smoke. Curr. Pharm. Des. 11, 2199-2208 (2005).

6 Ambrose JA, Barua RS. The pathophysiology of cigarette smoking and cardiovascular disease. An update. J. Am. Coll. Cardiol. 43(10), 1731-1737 (2004).

7 Leone A. Relation between coronary lesions and cigarette smoking of subjects deceased from acute myocardial a major cardiovascular risk factor. Thus smoking leads, in time, to the occurrence of the same cardiovascular disease, but with different degrees of changes. These are dependent on several factors such as number of cigarettes smoked, duration of exposure and health characteristics of the individual, among others. However, in pathological findings [19-21], when the experimental procedures are the same and well standardized, the cardiovascular damage from smoking is always reproducible in its features.

Finally, it is worth noting that cigarette smoking itself is able to cause a toxic disease whose etiologic factor should be considered the tobacco with its chemical compounds [22].

It is the author's personal opinion that a real link of cause-effect may certainly be identified between cigarette smoking and cardiovascular lesions, primarily ischemic heart disease and endothelial dysfunction, allowing tobacco smoke to be undoubtedly considered an etiologic factor of cardiovascular disease.

\section{Financial \& competing interests disclosure}

The author has no relevant affiliations or financial involvement with any organization or entity with a financial interest in or financial conflict with the subject matter or materials discussed in the manuscript. This includes employment, consultancies, honoraria, stock ownership or options, expert testimony, grants or patents received or pending, or royalties.

No writing assistance was utilized in the production of this manuscript.

\section{Open access}

This work is licensed under the Creative Commons Attribution 4.0 License. To view a copy of this license, visit http://creativecommons.org/licenses/by/4.0/

infarction. A histopathological study. J. Cardiobiol. 2(2), 1-5 (2014).

8 Lee PN, Chamberlain J, Alderson M. Relationship of passive smoking to risk of lung cancer and other smoking-associated diseases. Br. J. Cancer 54, 97-105 (1986).

9 Leone A. Relationship between cigarette smoking and other coronary risk factors in atherosclerosis: risk of cardiovascular disease and preventive measures. Curr. Pharm. Des. 9 , 2417-2423 (2003).

10 Sen S, Peltz C, Beard J, Zeno B. Recurrent carbon monoxide poisoning from cigarette smoking. Am. J. Med. Sci.340(5), 427-428 (2010).

11 Leone A, Giannini D, Bellotto C, Balbarini A. Passive smoking and coronary heart disease. Curr. Vasc. Pharmacol. 2, 175-182 (2004).

12 Kjeldsen K, Thomsen HK, Astrup P. Effects of carbon monoxide on myocardium: ultrastructural changes in rabbits after moderate, chronic exposure. Circ. Res. 34, 339-348 (1974). 
13 Messner B, Bernhard D. Smoking and cardiovascular disease: mechanisms of endothelial dysfunction and early atherogenesis. Arterioscler. Thromb. Vasc. Biol. 34(3), 509-515 (2014).

14 Leone A. Toxics of tobacco smoke and cardiovascular system: from functional to cellular damage. Curr. Pharm. Des. 21, 4370-4379 (2015).

15 Leone A, Landini L. Vascular pathology from smoking: look at the microcirculation. Curr. Vasc. Pharmacol. 11(4), 524-530 (2013).

16 Stefanidis C, Vlachopopoulos C, Tsiamis E et al. Unfavorable effects of passive smoking in aortic function in men. Ann. Intern. Med. 128(6), 426-434 (1998).

17 Leone A. Morphological alterations of the heart and blood vessels from tobacco smoke: the steps of the damage. J Cardiol. Ther. 2(4), 355-359 (2015).
18 Glantz SA, Parmley WW. Passive smoking and heart disease: epidemiology, physiology, and biochemistry. Circulation 83, 1-12 (1991).

19 Leone A. Passive smoking exposure and cardiovascular health. In: Handbook of Cardiovascular Research. Brataas J, Nanstveii V (Eds.). Nova Science Publishers, Inc. NY, USA, 81-111 (2009).

20 Lough J. Cardiomyopathy produced by cigarette smoke. Ultrastructural observations in guinea pigs. Arch. Pathol. Lab. Med. 102, 377-380 (1978).

21 Leone A, Bertanelli F, Mori L, Fabiano P, Bertoncini G. Ventricular arrhythmias by passive smoke in patients with preexisting myocardial infarction. J. Am. Coll. Cardiol. 3, 256A (1992).

22 Leone A, Landini L, Leone A. What is tobacco smoke? Sociocultural dimensions of the association with cardiovascular risk. Curr. Pharm. Des. 16, 2510-2517 (2010). 